\title{
Autonomous Sensory Meridian Response as an Intervention to Autism Spectrum Disorder
}

\author{
Ariana Galante ${ }^{1 *}$ and Nafees Alam ${ }^{2}$ \\ ${ }^{1}$ Researcher, College of Staten Island, USA \\ ${ }^{2}$ Researcher, Yeshiva University, USA
}

Submission: August 24, 2019; Published: September 10, 2019

*Corresponding author: Ariana Galante, Researcher, College of Staten Island, New York, USA

\begin{abstract}
Studies pertaining to Autonomous Sensory Meridian Response (ASMR) highlight the need for further research on ASMR and autism, paving the way for an increase in interventions for Autism Spectrum Disorder (ASD). The therapeutic use of ASMR videos provide a significant improvement in mood and behavioral development. ASMR allows audiences to reap the benefits of soothing and calming sensations. Using ASMR as audiovisual therapy can serve as a notable component to early intervention, alleviating symptoms associated with meltdowns and struggles of emotional self-regulation. Accessibility is important, as are pre-assessments for Misophonia and other potential factors that may limit the benefits of ASMR. The creation of an app that has videos of ASMR readily available can allow quick access when needed. In addition, Spotify, YouTube, and iTunes can have ASMR playlists and channels, classifying them as a genre along with more online forums discussing this topic to promote awareness. The advancement of ASMR through further research can potentially benefit the autistic community with its vast array of possibilities. ASMR may be the next step in promoting self-care in assisting individuals diagnosed with ASD.
\end{abstract}

Keywords: Autonomous Sensory Meridian Response (ASMR); Autism Spectrum Disorder (ASD); Early intervention; Misophonia.

\section{Introduction}

Children on the autism spectrum can benefit, both cognitively and emotionally, from advancements in early intervention. Autonomous Sensory Meridian Response (ASMR) can contribute to the aggregation of therapeutic practices geared toward the development of autistic individuals. ASMR is generally a response to visual and/or auditory stimuli that invokes tingling or static-like sensations on the scalp, moving down the back of the neck and upper-spine [1]. In theory, ASMR may be beneficial for behavioral development, serving as audiovisual therapy for individuals diagnosed with ASD (Autism Spectrum Disorder), because of its combination of physiological and psychological responses to visual and/or auditory stimuli.

\section{Background}

ASD is labeled as an intellectual disability, impacting cognitive abilities. However, not all cases are the same. ASD is categorized as a lifelong developmental disorder that affects communication and behavior. It is a "spectrum" due to its wide variation and severity of symptoms found in individuals with this diagnosis and alters a person's ability to function in daily life. ASD can be diagnosed at any age and does not isolate itself to one subgroup, and it impacts all ethnicities and economic groups [2]. Training in communication, as well as behavioral therapy, can help people with ASD improve their cognitive and behavioral limitations, as well as self-regulating successfully, and early intervention can assist these symptoms.

The kind of services available are physical and communicative skills, cognitive and adaptive skills, social and emotional skills, and sensory processing skills. Existing literature suggests the most used therapies for behavior regarding early intervention are ABA (Applied Behavior Analysis) Therapy, TEACCH (Treatment and Education of Autistic and Related Communication Handicapped Children), and PECS (Picture Exchange Communication System) [3]. The behavioral, psychological, and educational therapy provided with early intervention is significant for people with ASD to promote growth, considering the life-skills individuals possess when it comes to living independently, helping reduce taxing behaviors, building on strengths, and benefiting society, communication and language skills [2]. Autonomous Sensory Meridian Response has the potential to be a significant component of early intervention regarding emotional self-regulation tactics. 


\section{Psychology and Behavioral Science International Journal}

\section{What is Autonomous Sensory Meridian Response (ASMR)?}

Autonomous Sensory Meridian Response (ASMR) is an atypical sensory phenomenon in which individuals experience physical and psychological responses to auditory and/or visual stimuli, and the sensations are soothing due to the variety of its intimate, gentle nature. The stimuli mimics voices, sounds, behaviors, and moments often found in day to day life. The auditory stimuli, also known as "triggers," includes, but is not limited to, whispering, soft talking, and methodical sounds [1]. Some of these examples include the cycling of household items that derive various noises when tapped upon or used, which alternates binaural auditory stimuli [4]. Further examples of auditory stimuli are spoken sounds that are slow and gentle with monotone or increased pitch, vocal sounds such as chewing/mouth sounds, and object sounds such as tapping, scratching, cutting, eating, lip-smacking, crinkling, and even the syntax of spoken words. The visual stimuli, which are also known "triggers," include slow movements or eye contact with household items being used in a slow or methodical pace. ASMR is considered to be a non-threatening stimulus which is key to inducing relaxation. Although it is suggested that individuals may have different triggers, ASMR is associated with concentrated attention and intimacy.

The psychological and physiological sensations trigger feelings of deep and soothing relaxation, comfort, calmness, sleepiness, and peacefulness. Tingles, feelings of fuzziness or waves in the head, spine, neck, and throughout the rest of the body are the general sensations felt. Reports of ASMR magnify a state of "flow," which engages an individual in intense focus and diminishment of time concerning the optimal performance of the auditory and visual stimuli. According to a survey study of over 450 individuals with ASMR, it has been found that whispering, close up attention, and perceiving slow movements such as the brushing of hair conjured tingles in over half of its respondents [1].

ASMR has become an internet subculture to audiovisual therapy, the individuals who consume themselves in this online therapeutic culture are a community called "The Whisper Community" on social media platforms. This community uploads ASMR videos to YouTube, and then they are transferred over to other social media outlets such as Instagram, Reddit, and Snapchat. This online group of people create and post videos with the intention to exhibit relaxing, tranquil, lowgrade euphoric sensations. The ASMR community rely on the effective power of visual/auditory impressions to create an intimate space shared with the viewer/listener. This community uses high-quality microphones and cameras to give their audience the utmost placate experience during their mesmerizing rituals of gentle sounds and hand movements. Many of the ASMR content creators invest in expensive microphones, specifically to narrow in on sensitivity, low self-noise, and stereophonic capability. These areas of focus are vital to the ASMR community, attributed to triggers creating a three-dimensional effect by binaural recordings. The derivation of these binaural recordings serves a purpose of exhibiting hypnotic sounds, and the contrast between proximity and space is exacerbated [5].

\section{How Has ASMR Been Helpful with Other Issues?}

The physiological and psychological implications of ASMR are significant; there are numerous ASMR videos on social media platforms, totaling to about 13 million videos [6]. According to a recent study conducted from the University of Sheffield's Department of Psychology, participants had significantly greater reductions in heart rate (a decrease of 3.14 beats per minute) when watching ASMR videos as opposed to those who did not. Additionally, the same participants also expressed a necessary increase in positive emotions alongside feelings of social connection and relaxation. ASMR acquires conclusive evidence of mitigating the symptoms of anxiety, depression, stress, and insomnia. In another experiment conducted from the University of Sheffield's Department of Psychology it has been found that compared to non-ASMR participants, those who experience ASMR reported frequent tingling, heightened level of excitement and calmness, and a decrease in stress and sadness. Furthermore, Dr. Poerio from the University of Sheffield stated that ASMR videos do possess the relaxing effects reported from those who experience it, and the effects parallel with the physiological effects from stress-reduction techniques such as mindfulness and music [7].

It has also been noted that $80 \%$ of participants regarding a study conducted in 2015 reported an effect in their mood which was positive on average and those at high risk for depression reported that ASMR improved their mood [1]. 11\% of participants in a study conducted in 2017 stated they watch ASMR videos to reduce their anxiety and 70\% of participants in another study conducted in 2015 indicated that they watch ASMR videos to cope with stress [1,8]. 41\% of participants in a study conducted in 2017 stated that ASMR videos serve as an aid for falling asleep and in a study conducted in $2015,82 \%$ of participants watch ASMR videos to help them sleep [1,8]. According to ongoing research, the majority of participants diagnosed with anxiety or panic disorders report that ASMR helps them with their condition. Additionally, a majority of participants diagnosed with insomnia reported that ASMR helps them with their condition as well.

Viewers of the ASMR community vouch for its beneficial effects when commenting on ASMR videos posted to YouTube and other social networking sites, emphasizing their relaxation, reduction in anxiety, depression, sadness, insomnia, sleeplessness, and stress. Although there is sparse existing literature and scientific research to back up the reduction of symptoms, many viewers remain loyal and depend on the ASMR community to retrieve the interesting phenomenon experienced from ASMR videos. Currently, there are three published research articles, as cited above, that magnify the benefits of ASMR. Unfortunately, it will take more research and clinical studies before the clinical application of ASMR renders understood, though there are ongoing projects currently underway [9].

\section{How Can ASMR Serve as A Coping Mechanism for Autism?}

Toddlers and children diagnosed with ASD struggle with the assimilation of normative ways concerning behavior. The difficulties are 


\section{Psychology and Behavioral Science International Journal}

significant regarding social, sensory and intellectual adaptation; emotional management may be problematic. When a caretaker witnesses the triggers that send an autistic toddler or child into the beginning stages of a meltdown, it may be beneficial to navigate the symptoms as soon as possible to prevent the behaviors from escalating. ASMR can serve as a tool to help mitigate the severity of the negative, explosive behaviors, redirect, and benefit emotional self-regulation. Moreover, during the recovery stage following a meltdown, ASMR will soothe and aid calmness taking into account the necessary increase in positive emotions.

ASMR interventions can present as multifaceted approaches consisting of different auditory and visual stimuli to aid anxiety and stressors related to emotional self-regulation. The methods of ASMR convey relaxation and stress relief of overstimulation, which can range from loud noises to busy environments. Anxiety in those diagnosed with ASD lead to challenging behaviors and obtaining an understanding of the cycle of behaviors can help carry out ASMR appropriately. Understanding the cycle of behaviors can help alleviate escalation of minor events that trigger these behaviors. In opposition to negative, provoking stimuli, ASMR can provide a soothing environment that would be favorable to the advancement of centering oneself, calmness and relaxation.

Furthermore, noise canceling headphones and a computer or iPad displaying audio-visual stimuli from ASMR videos can promote positive stimulation along with the help of binaural recordings from high-quality microphones. This technique can serve as a crisis approach and a preventative measure, setting a valuable precedent for the day. Playing an ASMR video before or after a meltdown can be analogous to counting sheep, which is commonly known to relax an individual. Audiovisual therapy is beneficial for ASD considering it is a non-threatening stimulus and it can further benefit by recognizing the triggers and intervening appropriately. ASMR can help behavioral development and serve as a coping mechanism while promoting emotional self-regulation. Incorporating ASMR videos into intervention practices ultimately helps individuals diagnosed with ASD to react in less stigmatizing, and effective self-regulating ways.

\section{Gaps \& Limitations}

The lack of research studies covering the effects of ASMR remains a hinderance due to its general scarcity. More research into the topic is needed to gain a better understanding of ASMR and its benefits on the community. Existing literature, limited as it may be, conveys the potential benefits of ASMR as an added component for early intervention for individuals with ASD. However, ASMR may not be favorable for specific individuals who experience unusually severe sensory stimuli. Individuals with ASD experience overstimulation, and Misophonia may be a driving factor of this symptom. Sound sensitivity is prevalent as many people with ASD feel anxious and fearful of sound. Unfavorable physiological sensations are felt due to autonomic and/or behavioral responses to nonpreferred sounds [10].

Misophonia is a phenomenon that is characterized by intense emotional reactions, such as rage, anxiety, irritability, and disgust. Misopho- nics have reported experiencing these emotional reactions triggered by sounds of human origin such as chewing, sniffing, and lip-smacking sounds (Edelstein, 2013). A diagnosis of ASD may be comorbid with Misophonia. The sensitivity that individuals with Misophonia experience is generally triggered by a specific set of sounds. These triggers can be from day to day sounds such as those from cars, rain or airplanes, as well as sounds made by animals, which can potentially result in Misophonic reactions by certain individuals [11]. For those with Misophonia, ASMR may actually exacerbate emotional reactions as opposed to mitigating them.

\section{Conclusion \& Recommendations}

Despite the possibility of Misophonia wedging a gap between the positive effects of ASMR and autism, there may be alternative options for autistic, Misophonics to experience ASMR without the verbal triggers. Non-verbal videos can serve as a great substitute for verbal videos. Self-induced sounds serve as an appropriate coping mechanism for those diagnosed with Misophonia. The self-induced trigger sounds do not evoke panic and rage as opposed to sounds produced others [12].

Individuals on the spectrum that do not classify as misphonics can also create ASMR within themselves when experiencing extreme emotional stimuli and need the outlet to help them cope. Creating auditory stimuli that triggers ASMR can be beneficial, not just from the auditory and visual perspective, but from the tactile sensory perspective as well. Accessibility is significant, and allowing autistic people access to ASMR is important. The creation of an app that has videos of ASMR readily available can allow quick access when needed. In addition, Spotify, YouTube and iTunes can have ASMR playlists and channels, classifying them as a genre along with more online forums discussing this topic to promote awareness. The promotion of ASMR and the need for further research can promote and benefit the autistic community with its vast array of possibilities ready to be discovered and applied.

Ongoing studies pertaining to ASMR highlight the need for further research on ASMR and autism, paving the way for an increase in interventions for ASD. The therapeutic use of ASMR videos provide a significant improvement in mood and behavioral development. Experiencing ASMR when watching and listening to videos allows the audience to reap its benefits and applications of soothing and calming sensations. Using ASMR as audiovisual therapy can serve as a notable component to early intervention and coping mechanism, which alleviates and pacifies symptoms associated with meltdowns and struggles of emotional self-regulation. ASMR may be the next step in promoting self-care and assisting individuals diagnosed with ASD.

\section{References}

1. Barratt EL, Davis NJ (2015) Autonomous Sensory Meridian Response (ASMR): A flow-like mental state. PeerJ 3.

2. The National Institute of Mental Health (NIMH) is the lead federal agency for research on mental disorders. National Institute of Mental Health.

3. Bajko A (2018) The Importance of Early Diagnosis and Intervention for Children with Autism Spectrum Disorder. 
4. Smith SD, Fredborg BK, Kornelsen J (2016) An examination of the default mode network in individuals with autonomous sensory meridian response (ASMR) Soc Neurosci 12(4): 361-365.

5. Garro D (2017) Autonomous Meridian Sensory Response - From Internet Subculture to Audiovisual Therapy. ScienceOpen.

6. Brain tingles: First study of its kind reveals physiological benefits of ASMR (2018). Science Daily.

7. Poerio GL, Blakey E, Hostler TJ, Veltri T (2018) More than a feeling: Autonomous sensory meridian response (ASMR) is characterized by reliable changes in affect and physiology. Plos One 13(6).

8. Mcerlean AB, Banissy MJ (2017) Assessing Individual Variation in Personality and Empathy Traits in Self-Reported Autonomous Sensory Meridian Response. Multisensory Research 30(6): 601-613.
9. Health Benefits of ASMR (2019) ASMR University.

10. Stiegler LN, Davis R (2010) Understanding Sound Sensitivity in Individuals with Autism Spectrum Disorders. Focus on Autism and Other Developmental Disabilities 25(2): 67-75.

11. Cavanna AE, Seri S (2015) Misophonia: Current perspectives. Neuropsychiatr Dis Treat 11: 2117-2123.

12. Edelstein M, Brang D, Rouw R, Ramachandran VS (2013) Misophonia: Physiological investigations and case descriptions. Frontiers in Human Neuroscience 7.

\section{Your next submission with Juniper Publishers will reach you the below assets}

- Quality Editorial service

- Swift Peer Review

- Reprints availability

- E-prints Service

- Manuscript Podcast for convenient understanding

- Global attainment for your research

- Manuscript accessibility in different formats ( Pdf, E-pub, Full Text, Audio)

- Unceasing customer service

Track the below URL for one-step submission https://juniperpublishers.com/online-submission.php 